\title{
Desarrollo de una bebida mixta tipo néctar con cápsulas de Aloe vera (L.) Burm. f. y vitamina C
}

\section{Development of a nectar-type mixed drink with Aloe vera (L.) Burm. f. and vitamin $C$ capsules}

\author{
Diana Carolina Corzo-Barragán; Felipe Salcedo Galán²; Ricardo Arturo Pacheco ${ }^{3}$
}

1'Ing. Agroindustrial, Especialista en Gestión de Proyectos, Magister en Diseño de Productos y Procesos. Investigadora Jardín Botánico de Bogotá. Av. Calle 63 No. 68-95, Bogotá D.C., Colombia, e-mail: dcorzo@jbb.gov.co, Dhttps://orcid.org/0000-0001-7282-9289

${ }^{2}$ Ing. Químico Ph.D., Materials Science and Engineering. Profesor asistente, Departamento de Ingeniería Química. Universidad de Los Andes, Grupo de Diseño de Productos y Procesos (GDPP), Dhttps://orcid.org/0000-0002-7039-6448

${ }^{3}$ Ing. Agrónomo, Esp. en fruticultura de clima frío. Investigador Jardín Botánico de Bogotá, (Dhttps://orcid.org/0000-0001-7447-870X

Cómo citar: Corzo-Barragán, D.C.; Salcedo Galán, F.; Pacheco, R.A. 2019. Desarrollo de una bebida mixta tipo néctar con cápsulas de Aloe vera (L.) Burm. f. y vitamina C. Rev. U.D.C.A Act. \& Div. Cient. 22(1):e1180. https://doi.org/10.31910/rudca.v22.n1.2019.1180

Artículo de acceso abierto publicado por Revista U.D.C.A Actualidad \& Divulgación Científica bajo una licencia Creative Commons CC BY-NC 4.0

Recibido: Junio 18 de 2018

Aceptado: Marzo 6 de 2019

\section{RESUMEN}

El mercado de productos naturales, a nivel alimenticio, medicinal e industrial, se ha incrementado notablemente, promoviendo la innovación e investigación y el uso de materias primas vegetales y animales. La presente investigación tuvo como objetivo diseñar un néctar, con una combinación de matrices de frutas y de vegetales, como uva, espinaca, mora, agraz, yacón, enriquecida con cápsulas de Aloe vera y vitamina C. Se plantearon tres formulaciones, variando la cantidad de ingredientes en la mezcla; se seleccionó el tratamiento más aceptable, mediante evaluación sensorial en panel no entrenado. Todos los néctares tuvieron una buena aceptación organoléptica; no obstante, el $50 \%$ de los panelistas mostraron preferencias por el tratamiento 1 , que contenía $13 \%$ de uva, 4\% de espinaca, 3\% de agraz y mora, $2 \%$ de yacón; el porcentaje restante correspondió a la cantidad de agua y azúcar empleada en la formulación. Al tratamiento con mayor aceptación (T1), se le analizaron los atributos físico químicos, mostrando un $\mathrm{pH}$ de $2,96,12,11^{\circ} \mathrm{Brix}$, color, olor y apariencia normales y estables; asimismo, se evaluó la presencia de bacterias mesófilas, hongos y levaduras, E. coli y Salmonella. Con el fin de enriquecer el producto, se elaboraron cápsulas de A. vera y vitamina $C$, para ser incorporadas en el néctar. Este tipo de producto promueve el consumo de frutas y de vegetales en alimentos procesados de uso frecuente, como los néctares.

Palabras clave: aceptabilidad; alimentos sanos; encapsulación; características organolépticas; productos nuevos. CAB Thesaurus.

\section{ABSTRACT}

The market of natural products at a nutritional, medicinal and industrial level, has increased remarkably, promoting innovation and research and the use of vegetable and animal raw materials. The objective of this research was to design a nectar, with a combination of fruit and vegetable matrices such as grape, spinach, blackberry, blueberry, yacon, enriched with Aloe vera capsules and vitamin $\mathrm{C}$. Three formulations were proposed varying the number of ingredients in the mixture; the most acceptable treatment was selected through sensory evaluation in an untrained panel. All the nectars had a good organoleptic acceptance, however, $50 \%$ of the panelists showed preferences for the treatment 1, it contained 13\% of grape, $4 \%$ of spinach, $3 \%$ of blueberry and blackberry, $2 \%$ 
of yacon, the remaining percentage corresponded to the amount of water and sugar used in the formulation. To the treatment with greater acceptance (T1) the physical-chemical attributes were analyzed, showing a $\mathrm{pH}$ of $2.96 ; 12.11^{\circ} \mathrm{Brix}$, color, smell and appearance normal and stable, likewise the presence of mesophilic bacteria, fungi and yeasts, E. coli and Salmonella was evaluated. In order to enrich the product, capsules of $A$. vera and vitamin $\mathrm{C}$ were prepared to be incorporated into the nectar. This type of product promotes the consumption of fruits and vegetables in processed foods of frequent use such as nectars.

Keywords: acceptability; healthy foods; encapsulation; organoleptic characteristics; new products.

\section{INTRODUCCIÓN}

Si bien, desde la antigüedad, las plantas fueron usadas por el hombre con fines alimenticios, medicinales e industriales, es en la modernidad, donde se ha promovido el desarrollo de productos con ingredientes naturales, dada la tendencia generalizada por preferir alimentos que empleen materias primas de fuentes naturales con actividades biológicas, por el aumento de las enfermedades crónicas no transmisibles, como el cáncer, la obesidad, la diabetes, la hipertensión, entre otras (OMS, 2011), lo cual, ha permitido que el mercado de productos naturales haya incrementado.

En ese sentido, los productos vegetales, tales como frutas y hortalizas, son una opción viable, debido a su alto potencial nutricional y terapéutico, gracias a la presencia de diferentes compuestos bioactivos de interés. En efecto, Araya et al. (2006) reportan que el consumo de frutas y vegetales ha sido asociado, con una menor incidencia, a padecer enfermedades y mortalidad por diferentes causas, dada las propiedades que estos poseen. Esto representa un gran reto para las empresas de alimentos y bebidas, en el diseño de productos que, sin duda, tendrá un impacto en la salud; la combinación de matrices mixtas de frutas y verduras, se pueden emplear en distintas presentaciones, con una escala de procesamiento, que abarca desde la transformación primaria hasta tener diversos productos procesados, siendo el néctar una opción viable; según la norma técnica Colombiana NTC 5468 (ICONTEC, 2007), los néctares son definidos como una bebida elaborada con pulpa de una o varias frutas, adicionando agua y azúcar.

En el presente estudio, se usaron especies vegetales que poseen actividad antioxidante (Plazas \& Pacheco, 2011; Rockenbach et al. 2011), como la uva isabella (Vitis labrusca L.) y el agraz (Vaccinium meridionales $\mathrm{Sw}$ ), que coadyudan en el tratamiento de la hipoglicemia (Corantioquia, 2009); la mora (Rubus glaucus Benth), importante en el tratamiento de enfermedades infecciosas de la garganta (García, 1975); la espinaca (Spinacia oleracea L.), rica en minerales y vitaminas (Araya et al. 2006) y la sábila (Aloe vera (L.) Burm. f.), caracterizada por la presencia de compuestos fenólicos, además de sus propiedades antibacterianas, antiviral y contenido de vitaminas y de minerales (Vega et al. 2005). Adicionalmente, se usó Yacón (Samallanthus sonchifolius (Poepp.) H. Robinson), que almacena frutooligosacaridos, bajos en calorías y no elevan el nivel de glucosa en la sangre (Seminario et al. 2003).

Con el fin innovar y mejorar la calidad sensorial en el producto, se planteó la microencapsulación del $A$. vera, junto con vitamina $\mathrm{C}$, por ser antioxidante y contribuir al mantenimiento del material intercelular (Bastías \& Cepero, 2016); la encapsulación permite que los materiales puedan ser sometidos a diferentes condiciones de proceso sin afectar sus características, igualmente, mejora las propiedades organolépticas (Yañez et al. 2002).

Con base en lo anterior, la presente investigación buscó aprovechar las características de las materias primas mencionadas, para la formulación de una bebida mixta tipo néctar, enriquecida con cápsulas de $A$. vera y vitamina $\mathrm{C}$, elaborada sin aditivos ni conservantes.

\section{MATERIALES Y MÉTODOS}

Procesamiento de la materia prima. Las muestras fueron adquiridas en el mercado local y sometidas a operaciones unitarias de recepción, pesaje, lavado y desinfección, con una solución de hipoclorito de sodio a $200 \mathrm{ppm}$, escaldadas a $80^{\circ} \mathrm{C}$, por 3 minutos y despulpadas de manera manual; las pulpas, se analizaron por el método potenciométrico A.O.A.C. 981.12/1990, para medir el $\mathrm{pH}$, usando un $\mathrm{pH}$-metro de contacto marca Fieldscout-soilstik; los sólidos solubles, se analizaron por el método refractométrico A.O.A.C 932.12/1990, usando refractómetro digital-sper scientific, mediciones que se realizaron por triplicado. En esta etapa, se determinaron los rendimientos del proceso y, con las pulpas, se elaboraron los distintos tratamientos.

Formulaciones. Se plantearon tres tratamientos, en donde se dejó constante la cantidad de agua, que correspondió a una proporción 1:3 (v/v) siendo 1 la pulpa y 3 agua; se adicionó la cantidad de sacarosa necesaria, para ajustar la mezcla a $12^{\circ}$ Brix y, el $25 \%$ restante, correspondió a la pulpa de fruta combinada (uva, espinaca, mora, agraz y yacón), en las proporciones indicadas en la tabla 1.

Para la elaboración del néctar, se mezclaron las matrices, de acuerdo con lo planteado en cada formulación; posteriormente, se pasteurizaron por tres minutos y se envasaron en caliente en frascos de vidrio con capacidad de $200 \mathrm{~mL}$, con tapa hermética, tipo twist off, previamente esterilizados. Finalmente, se realizó un choque térmico; en este punto, se adicionaron 10 cápsulas de $A$. vera por cada envase, las cuales, quedaron en suspensión. Los néctares fueron almacenados a $8^{\circ} \mathrm{C}$, hasta su uso.

Prueba de percepción. Con el objetivo de seleccionar el tratamiento con mayor aceptación, se realizó una evaluación sensorial (Ávilade Hernández \& González-Torrivilla, 2011) con un panel no entrenado de 20 personas, donde evaluaron hedónicamente los siguientes atributos: color, aroma, dulzor, acidez, sabor, aceptación y preferencia (Figura 1). 
Tabla 1. Composición de la formulación de los néctares.

\begin{tabular}{|l|c|c|c|}
\hline \multicolumn{1}{|c|}{ Matriz } & Tratamiento 1 & Tratamiento 2 & Tratamiento 3 \\
\hline Agua & $66 \%$ & $66 \%$ & $66 \%$ \\
\hline Pulpa uva & $13 \%$ & $10 \%$ & $15 \%$ \\
\hline Pulpa espinaca & $4 \%$ & $10 \%$ & $2 \%$ \\
\hline Pulpa mora & $3 \%$ & $3 \%$ & $2 \%$ \\
\hline Pulpa agraz & $3 \%$ & $2 \%$ & $1 \%$ \\
\hline Pulpa yacón & $2 \%$ & 0 & \\
\hline Endulzante (azúcar blanca) & c.s.p. lograr $12^{\circ}$ Brix & \\
\hline De cada tratamiento se realizaron 5 réplicas de $200 \mathrm{~mL}$ & \\
\hline
\end{tabular}

Expresados en: $\%(\mathrm{v} / \mathrm{v})$

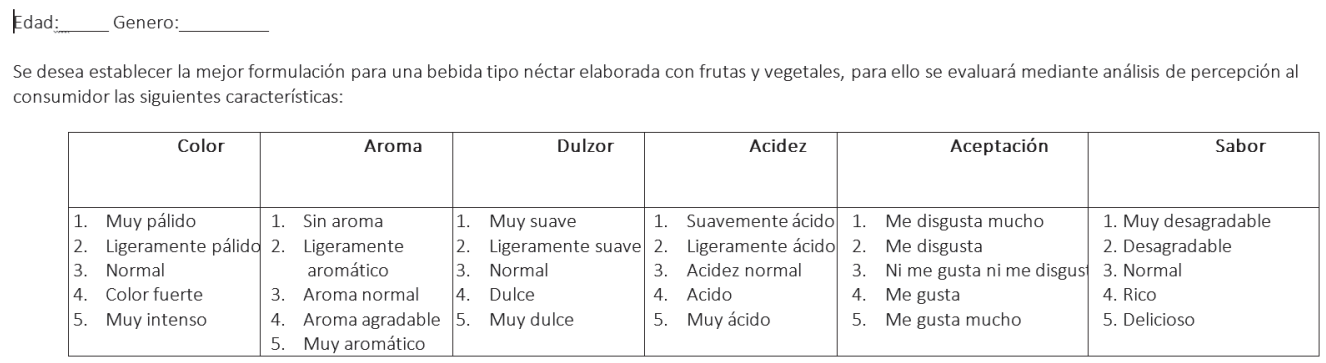

Diligencia la siguiente tabla de acuerdo a la información anterior, recuerde tomar entre cada muestra un sorbo de agua.
\begin{tabular}{|l|l|l|l|}
\hline Parámetroanalizado & T1 & T2 & \\
\hline Color & & & \\
\hline Aroma & & & \\
\hline Dulzor & & & \\
\hline Acidez & & & \\
\hline Sabor & & & \\
\hline
\end{tabular}

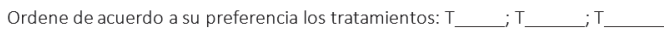

Observaciones:

Figura 1. Formulario de encuesta aplicado en las pruebas organolépticas.

En la figura, se observa el formato de evaluación, que se basó en el modelo de encuesta pre-establecido por Arango (1992). Las muestras, se dispusieron en copas transparentes en volúmenes de $20 \mathrm{~mL}$, a temperatura de refrigeración y fueron presentadas simultáneamente, debidamente codificadas.

Realización de las cápsulas y caracterización. Para la obtención del gel de A.vera, se procesó la penca de manera manual, retirando la cáscara; el gel fue cortado en trozos y licuado hasta su completa dilución y se le adicionaron $100 \mathrm{mg}$ de vitamina $\mathrm{C}$, en gotas por cada /100mL de sábila; se probaron concentraciones al 10 y 100\% de la mezcla de sábila y de vitamina $\mathrm{C}(\mathrm{v} / \mathrm{v})$, para ser encapsuladas; en el caso de la concentración al 10\%, el restante fue completado con agua potable, a esta mezcla fue incorporado, de manera gradual, como agente encapsulante, alginato de sodio grado alimenticio, en tres concentraciones, al 0,5, 1 y $2 \% \mathrm{p} / \mathrm{v}$; la mezcla se agito a $600 \mathrm{rpm}$, usando un impeller de propela de acero inoxidable, por 30 minutos; en este proceso de mezclado, se evaluaron dos temperaturas ambiente 19 y $30^{\circ} \mathrm{C}$. Paralelamente, se preparó una solución de cloruro de calcio al 0,1M y 3\% p/v, a temperatura de $40^{\circ} \mathrm{C}$ y se mezcló con agitador magnético, a 200rpm, durante 15 minutos. La solución polimérica sábila, vitamina $\mathrm{C}$ y alginato, se contuvo en jeringa de $10 \mathrm{~mL}$ y se incorporó por goteo en un Beaker, que contenía la solución de cloruro de calcio, a una altura de caída de $5 \mathrm{~cm}$; las capsulas, se dejaron en la solución de cloruro de calcio por 15 minutos y, posterior a ello, se filtraron con tamiz, para luego ser lavadas con agua destilada; este procedimiento, se realizó según las metodologías descritas por Castillo et al. (2017), con modificaciones en el instrumento de mezclado, tiempos en el proceso y concentración de cloruro de calcio; también, se siguió la metodología descrita por Parra (2014). A las cápsulas obtenidas, se caracterizaron midiendo el peso con balanza analítica Ohaus; con medición estereoscópica, se analizó la morfología con el software de microscopio Motic; además, se realizaron pruebas de penetración para determinar la firmeza de las cápsulas, con un penetrómetro modelo Fht200 Extech Instruments; todas las mediciones se realizaron por triplicado. 
Caracterización producto final. Al néctar con mayor preferencia, se evaluó por triplicado el $\mathrm{pH}$, por el método potenciométrico AOAC 981.12/21990; sólidos solubles totales, por el método AOAC 932.12/ 1990; al séptimo día de elaborado el néctar, se hicieron análisis microbiológicos, mediante la técnica de recuento en placa de profundidad, donde evaluó la presencia de bacterias mesófilas, hongos y levaduras, E. Coli y Salmonella; las muestras se analizaron por triplicado.

Con analizador óptico Turbiscan Lab, se evaluó, por triplicado, la estabilidad de la muestra o el comportamiento del néctar, en cuanto a la sedimentación/clarificación, durante 1.800 segundos.

\section{RESULTADOS Y DISCUSIÓN}

Procesamiento de la materia prima. Los rendimientos del despulpado se expresan en la tabla 2, que oscilaron entre el 15 y $40 \%$. En la misma tabla, se puede observar la caracterización de las pulpas, encontrando que estos valores concuerdan con Ayala et al. (2015), quienes reportaron, para la pulpa de mora, un $\mathrm{pH}$ entre el $2,64-2,68$ y $8^{\circ} \mathrm{Brix}$; en general, los valores reportados en las pulpas, para $\mathrm{pH}$, se encuentran dentro del rango de la mayoría de frutas tropicales (Torrenegra et al. 2016).
Prueba de percepción. De los panelistas evaluados+, el 30\% fueron hombres y el $70 \%$ mujeres, entre los $20-45$ años de edad.

De los tratamientos evaluados, el que presentó mayor preferencia fue el T1, que contenía 13\% de uva, $4 \%$ de espinaca, $3 \%$ de agraz y mora, $2 \%$ de yacón, además de la cantidad de agua y azúcar fijada en la formulación, con un $50 \%$ de favorabilidad, seguido del T3 y T2, con un 30 y $20 \%$. En cuanto a la aceptación de los productos estuvo en un rango de me gusta a me gusta mucho, en los tres tratamientos evaluados. Los néctares que usan mezcla, son bien recibidos entre los consumidores, tal como lo reportan Rojas et al. (2012); asimismo, en el estudio realizado por Pomareda (2017) mostró aceptación de un néctar de maracuyá, con sustitución parcial de pulpa de brócoli, en población infantil.

El análisis de percepción permitió concluir que el color en todos los tratamientos estuvo en el rango de normal o fuerte; el aroma, se expresó mejor en el T1, teniendo un rango normal, mientras que los tratamientos T2 y T3 fueron catalogados como ligeramente aromáticos; el dulzor en todos los tratamientos fue definido como normal, teniendo mejores resultados para el T2. En cuanto a la acidez y dada las características del producto final, este parámetro fue clasificado como ligeramente ácido, siendo el menos ácido el

Tabla 2. Caracterización de pulpas empleadas en las formulaciones.

\begin{tabular}{|l|c|c|c|c|c|}
\hline & Uva & Agraz & Espinaca & Yacón & Mora \\
\hline $\mathrm{pH}$ & 3,14 & 2,86 & 6,38 & 5,50 & 2,68 \\
\hline Sólidos solubles totales $\left({ }^{\circ} \mathrm{Brix}\right)$ & 12,7 & 8,2 & 1 & 6,1 & 6,2 \\
\hline Rendimiento de despulpado $(\%)$ & 32,30 & 23,40 & 15,74 & 15 & 40 \\
\hline
\end{tabular}

T2. Las preferencias mostraron, en cuanto a la aceptación de los productos, que estuvo en un rango de me gusta a me gusta mucho, en los tres tratamientos, con valores más altos para el T1; el sabor fue descrito, normal para el T2 y rico para el T1 y T3.

Realización de las cápsulas y caracterización. Las concentraciones de sábila evaluadas no afectaron la formación de las cápsulas; sin embargo, para obtener un producto con mejores características, desde el punto de vista funcional para el néctar, se decidió usar sábila al $100 \%$, para la realización de las mismas. Se pudo establecer que la mejor concentración de alginato de sodio fue del $2 \% \mathrm{w} / \mathrm{v}$. Observar los resultados con las diferentes concentraciones de alginato permite definir, de acuerdo con las características del material a encapsular, cuál es la concentración más apropiada; de las dos temperaturas analizadas en el proceso de mezcla del $A$. vera, vitamina $\mathrm{C}$ y alginato, la de $30^{\circ} \mathrm{C}$ fue la más favorable, mientras que, para el cloruro de calcio, se seleccionó la concentración al 3\% pv. Las cápsulas presentaron tamaño redondeado y peso uniforme de $0,08 \mathrm{~g}$, con un largo de $2,79 \mathrm{~mm}$ y ancho $2,86 \mathrm{~mm}$ y una firmeza de $0,54 \mathrm{Kg} / \mathrm{cm}^{2}$; en la figura 2 , se observa la morfología.
Caracterización producto final. El T1 presento un $\mathrm{pH}$ de 2,96; $12,11^{\circ}$ Brix y características organolépticas olor y apariencia normales y estables; estos valores están dentro del rango obtenido en néctar de zarzamora, por Valencia \& Guevara (2013) y Rojas et al. (2012).

Los resultados del análisis microbiológico fueron: para bacterias mésofilas < $10 \mathrm{UFC} / \mathrm{mL}$; para hongos y levaduras $>10 \mathrm{UFC} / \mathrm{mL}$; para E. coli $1 \times 1 \times 10^{2} \mathrm{UFC} / \mathrm{mL}$ y spara almonella $<10 \mathrm{UFC} / \mathrm{mL}$. Estos datos se encuentran dentro de los rangos permisibles, por la resolución número 7992 de 1991 (MinSalud, 1991).

Con el analizador óptico Turbiscan, se evidenció fenómeno de cremado, en la zona media y alta (Figura 3), común en los néctares, ya que los sólidos tienden a suspenderse y, al momento de agitar, las partículas empiezan a unirse u homogenizarse. Este proceso de desestabilización es uno de los más comunes, debido a la diferencia de densidades del sistema.

El néctar diseñado, se puede considerar un alimento saludable, dada las características proporcionadas en la mezcla de pulpas 


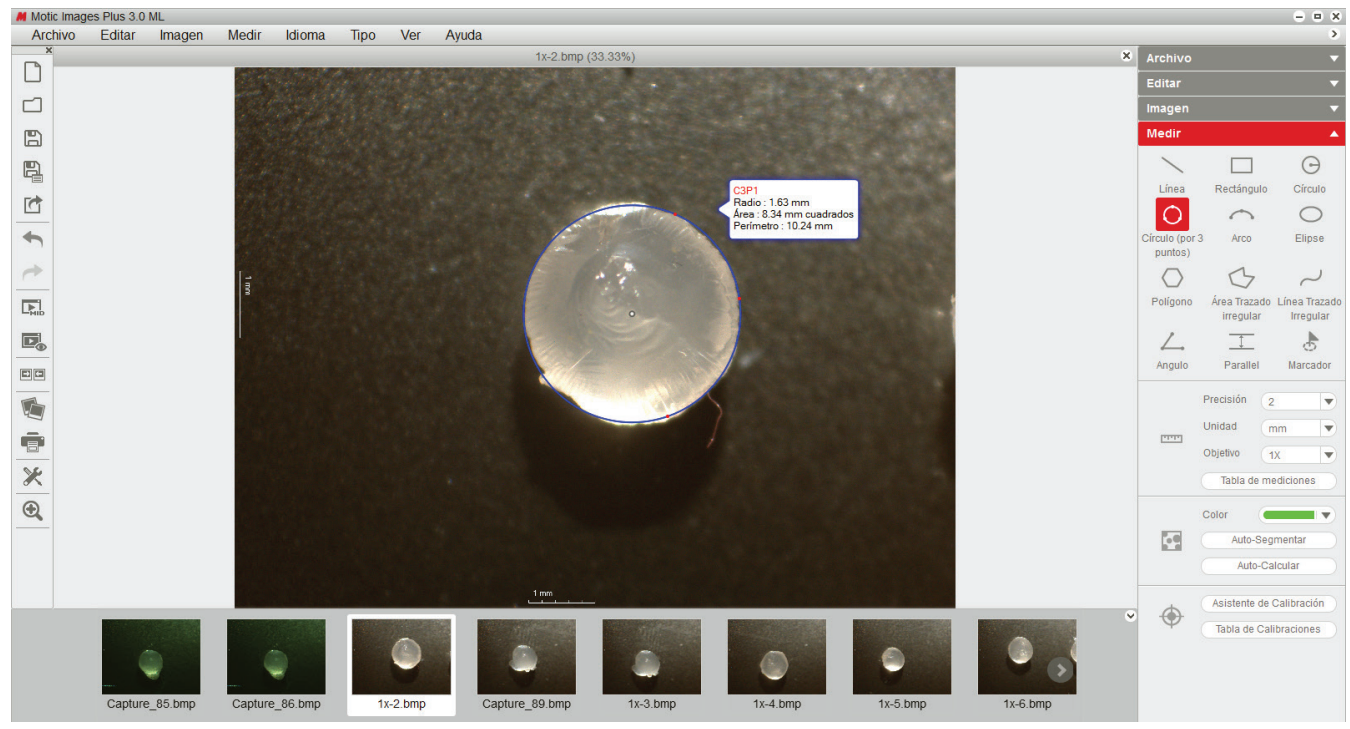

Figura 2. Análisis de la morfología de las cápsulas.

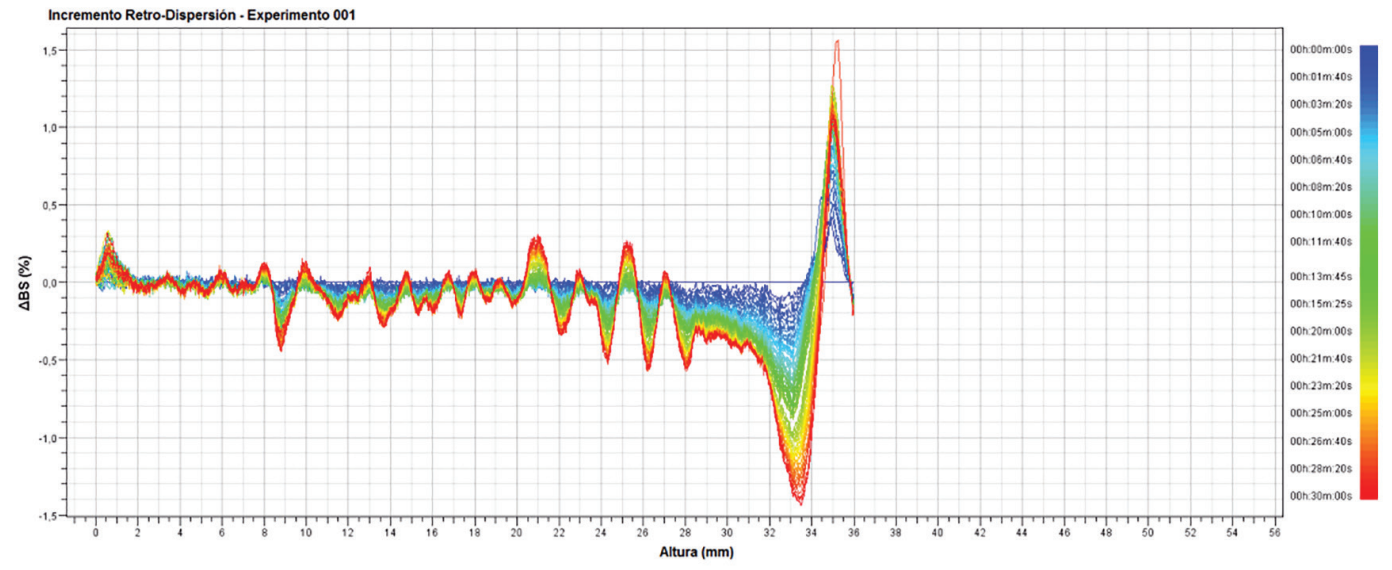

Figura 3. Análisis instrumental de estabilidad en el néctar.

seleccionadas, así como la incorporación de cápsulas de $A$. vera y vitamina $\mathrm{C}$, convirtiéndose en una bebida con alto valor agregado, que mostró buena aceptación entre los consumidores. Es importante, ya tendiendo definido el diseño de producto, realizar una caracterización nutricional, así como la determinación del potencial antioxidante en el producto final.

Conflicto de intereses: El manuscrito fue preparado y revisado con la participación de todos los autores, quienes declaramos que no existe conflicto de intereses que ponga en riesgo la validez de los resultados presentados. Financiación: Este estudio fue financiado por el programa de estímulos a la investigación Thomas van der Hammen en la convocatoria III- 2017.

\section{REFERENCIAS}

1. ARANGO, L.M. 1992. Evaluación sensorial. Manual de funcionamiento panel interno. Sabor, ciencia de alimentos Ltda.71p.

2. ARAYA, L.; ClAVIJO, H.; HERRERA, C. 2006. Capacidad antioxidante de frutas y verduras cultivados en Chile. Rev. Archivos Latinoamericanos de Nutrición (Venezuela). 56(4):361-365.

3. ASSOCIATION OF OFFICIAL ANALYTICAL CHEMISTSAOAC. 1990. Official methods of analysis. 932.12 Solids (Soluble) in fruits and fruit products. Refractometer Method.15th edition. Washington, DC. 
4. ASSOCIATION OF OFFICIAL ANALYTICAL CHEMISTSAOAC. 1990. Official methods of analysis. $981.12 \mathrm{pH}$ of Acidied Foods.15th edition. Washington, DC.

5. AYALA, L.C.; VALENZUELA, C.P.; BOHORQUEZ, Y. 2015. Caracterización fisicoquímica de mora de castilla (Rubus glaucus Benth) en seis estados de madurez. Biotecnología en el Sector Agropecuario y Agroindustrial. (Colombia). 11(2):10-18.

6. ÁVILA-DE HERNÁNDEZ, R.M.; GONZÁLEZTORRIVILLA, C.C. 2011. La evaluación sensorial de bebidas a base de fruta: una aproximación difusa. Universidad, Ciencia y Tecnología. (Venezuela). 15(60):171182. http://dx.doi.org/10.17268/sci.agropecu.2015.02.02

7. BASTÍAS, J.M.; CEPERO, Y. 2016. La vitamina C como un eficaz micronutriente en la fortificación de alimentos. Rev. Chilena de Nutrición. (Chile). 43(1):81-86. http://dx.doi. org/10.4067/S0717-75182016000100012

8. CASTILLO, S.L.; ALVARADO, J.M.; BAEZ, J.G.; MACÍAS, E.; RAMÍREZ-BACA, P.; CANDELAS-CADILLO, A.G.; GALLARDO, C.T. 2017. Diseño de microcápsulas de alginato con matriz prebiótica de aloe vera para la encapsulación de Lactobacillus plantarum. Investigación y Desarrollo en Ciencia y Tecnología de Alimentos. (México). 2:531-536.

9. CORPORACIÓN AUTÓNOMA REGIONAL DEL CENTRO DE ANTIOQUIA-CORANTIOQUIA. 2009. Conozcamos y usemos el mortiño (Colombia). Tercera edición. 28p.

10. GARCíA B., H. 1975. Flora Medicinal de Colombia, Botánica Médica. Tomo III. Talleres Editoriales de la Imprenta Nacional.

11. INSTITUTO COLOMBIANO DE NORMAS TÉCNICAS Y CERTIFICACIÓN-ICONTEC. 2007. Norma técnica colombiana 5468. 2007. Zumos (Jugos), néctares, purés (Pulpas) y concentrados de frutas.

12. MINISTERIO DE SALUD-MINSALUD. 1991. Resolución número 7992 de 1991. Elaboración, conservación y comercialización de Jugos, concentrados, néctares, pulpas, pulpas azucaradas y refrescos de frutas. Bogotá, Colombia, (21 de julio de 1991).

13. ORGANIZACIÓN MUNDIAL DE LA SALUD-OMS. 2011. Informe sobre la situación mundial de las enfermedades no transmisibles 2010. Resumen de orientación. Disponible desde Internet en http:/ / www.who.int/nmh/publications/ ncd_report_summary_es.pdf (con acceso 26/07/2017)
14. PARRA, R. 2014. Encapsulación de sábila (Aloe vera) y su efecto durante la incubación de yogur. Rev. Cultura Científica. (Colombia). 1:66-73.

15. PLAZAS, E.A.; PACHECO, R.A. 2011. Uso sostenible de especies vegetales del Distrito Capital y la región: potencial fitoquímico y de actividad biológica de especies altoandinas. Rev. Facultad Química Farmacéutica. (Colombia). 18(2):S1S344.

16. POMAREDA ANGULO, S.S. 2017. Obtención de néctar de Maracuyá (Passiflora eduiis) con sustitución parcial de pulpa de brócoli (Brassica olerácea) y su aceptación por niños en edad escolar. Informe final de investigación. Universidad Nacional Jorge Basadre Grohmann: 36p.

17. ROJAS, J.; GORDILLO, C.; GUERRERO, N.; IZÁZIGA, N.; LAGUNA, B.; LÁZARO, M. 2012. Efecto de la proporción de naranja (Citrus sinensis), papaya (Carica papaya) y piña (Ananas comosus) en la aceptabilidad sensorial de un néctar mixto. Agroindustrial Science (Perú). 2(2):132-138.

18. ROCKENBACH, I.I.; RODRIGUES, E.; GONZAGA, L.V.; CALIARI, V.; GENOVESE, M.I.; GONÇALVES, A.E.D.S.S.; FET'T, R. 2011. Phenolic compounds content and antioxidant activity in pomace from selected red grapes (Vitis vinifera L. and Vitis labrusca L.) widely produced in Brazil. Food Chemistry. 127(1):174-179. https://doi. org/10.1016/j.foodchem.2010.12.137

19. SEMINARIO, J.; VALDERRAMA, M.; MANRRIQUE, I. 2003. El yacón: fundamentos para el aprovechamiento de un recurso promisorio. Centro internacional de la papa (CIP), Universidad de Cajamarca, Agencia Suiza para el desarrollo y la cooperación. ( Perú): 60p

20. TORRENEGRA, A.; VILLALOBOS, L.; CASTELLAR, O.L.; LEÓN, E.A.; GRANADOS, C.; PAJARO, N.P.; SOLEDAD, I. 2016. Evaluación de la actividad antioxidante de las pulpas de Rubus glaucus B, Vaccinium floribundum $\mathrm{K}$ y Beta vulgaris L. Rev. Cubana de Plantas Medicinales. (Cuba). 21(4):1-8

21. VALENCIA, C.; GUEVARA, A. 2013. Elaboración de néctar de zarzamora (Rubus fructicosus L.). Scientia Agropecuaria. (Perú). 4(2):101-109. http://dx.doi.org/10.17268/sci. agropecu.2013.02.03

22. VEGA G., A.; AMPUERO C, N.; DÍAZ N, L.; LEMUS M., R. 2005. Aloe Vera (Aloe barbadensis Miller) as a component of functional foods. Rev. Chilena de nutrición. 32(3):208-214. http://dx.doi.org/10.4067/S0717-75182005000300005

23. YAÑEZ, J.; SALAZAR, J.; CHAIRES, L.; JIMENEZ, J.; MARQUEZ, M.; RAMOS, E. 2002. Aplicaciones biotecnológicas de la microencapsulación. Rev. Avance y Perspectiva. (México). 21:313-319. 